\title{
Conceptual Advances in the Default Space Model of Consciousness
}

\author{
Ravinder Jerath ${ }^{1 *}$, Connor Beveridge ${ }^{1}$, Michael Jensen ${ }^{2}$ \\ ${ }^{1}$ Charitable Medical Healthcare Foundation, Augusta, GA, USA \\ ${ }^{2}$ Augusta University, Augusta, GA, USA \\ Email: ^Rj605r@aol.com
}

How to cite this paper: Jerath, R., Beveridge, C. and Jensen, M. (2018) Conceptual Advances in the Default Space Model of Consciousness. World Journal of Neuros cience, 8, 254-269.

https://doi.org/10.4236/wjns.2018.82020

Received: March 2, 2018

Accepted: May 8, 2018

Published: May 11, 2018

Copyright (C) 2018 by authors and Scientific Research Publishing Inc. This work is licensed under the Creative Commons Attribution International License (CC BY 4.0).

http://creativecommons.org/licenses/by/4.0/

\section{(c) (i) Open Access}

\begin{abstract}
The default space model is a unified theory of consciousness that posits the brain and body together form the foundation of conscious experience that exists as a three dimensional internally generated simulation of reality termed the 3D dynamic default space. We have explored and developed the model in many publications and journals with a variety of academic specialties and its scope and concepts continue to broaden. In these publications, we have supported the concepts of the model through its ability to explain neuropsychological disorders, illusions, and everyday observations on consciousness. The model's foundations in which the thalamus serves as a central hub networked with the brain and body by continuous, fast, membrane potential oscillations have been greatly expanded since its initial publication which we review within this article. Profound leaps forward in our theory include the nature of lateral inhibition in sensory perception, the nature of sensory organs acting as "smart screens", and the correlation of respiration with mental atmosphere. Through reviewing the developing concepts expanding the theory since our major 2015 publication that laid the foundation of our theory, we hope to give readers a summarized update of where the theory currently stands in terms of its structure. We encourage readers to investigate these previous publications to gain further insight into our propositions. Through accurate models of consciousness, we may develop etiologies for countless neurological disorders, as well as improve treatments.
\end{abstract}

\section{Keywords}

Lateral Inhibition, Consciousness, Default Space, Respiration, Vision

\section{Introduction}

The brain may be explained as a "machine" that processes information and 
creates ideas. Somehow consciousness of some of this activity arises, albeit a small amount. We define consciousness simply in terms of the presence of experience. How is it that some brain activity is conscious while some is unconscious? Why is it that we feel as if we are a "ghost in a machine"? How could such a thing as consciousness exist in the first place? In order to even begin to answer these questions, an architecture of consciousness must be elucidated. Via the default space model, we propose such an architecture as well as provide insight into the mind-body problem which is the quandary of the relationship between mental states and brain states as well as the nature of the relationship between the conscious experience and the electro-chemical physiologies of the body [1].

The advances we have made in the theory provide insight into some of the top mysteries of neuroscience such as the true nature of sleep and baseline neural activity. Strong advancements of the default space theory include the explanation of previously described human physiology to illustrate the functional role of lateral inhibition in the sensory organs [2] [3], the role of graded membrane potentials and respiration in powering consciousness [4], the calibration of space and motion with photoreceptors [5], how we perceive the external world, and other developing concepts. The survey we provide in this article will allow those familiar with the foundations of the default space model to review how it has grown, however, those just learning of the model will be able to gain an understanding of its substance. We encourage readers to refer to our previous articles in which full explanations of the summaries we provide here can be found.

\section{The 3D Default Space: Theoretical Foundations}

Understanding the main focal points of the default space model will allow a deeper comprehension of the newer aspects of the model. The model describes how sensory information is processed, integrated, and filled-in within a $3 \mathrm{D}$ intra-personal space which is the backbone of consciousness [6] (Figure 1). This "naked", intra-personal space which becomes clothed by sensory qualia is the base form of the default space which we have termed the "Dark Space". This dark space is normally undetected by a person, and exists continuously, even when free of filled sensation [7]. This dark, neural-sensory-memory space is formed and maintained by the default mode network, resting state networks, and reticular activating system [8]. The external world is essentially simulated in this internal space in near real time [9]. All conscious experience, including dreaming and recollection of memories, occurs within this default space [4]. Because the default space replicates the physical nature of the environment, it provides optimal perception of and survival in such an environment. We assert this evolutionary benefit lead the original, neurological, consciousness producing mechanisms of animal ancestors utilize a default space [6]. Therefore, we assert the default space has its origins in the first conscious organisms.

The 3D Default space is maintained and formed from all the cells of the body [4], which are networked via afferent and efferent neurons [11], gap junctions, 


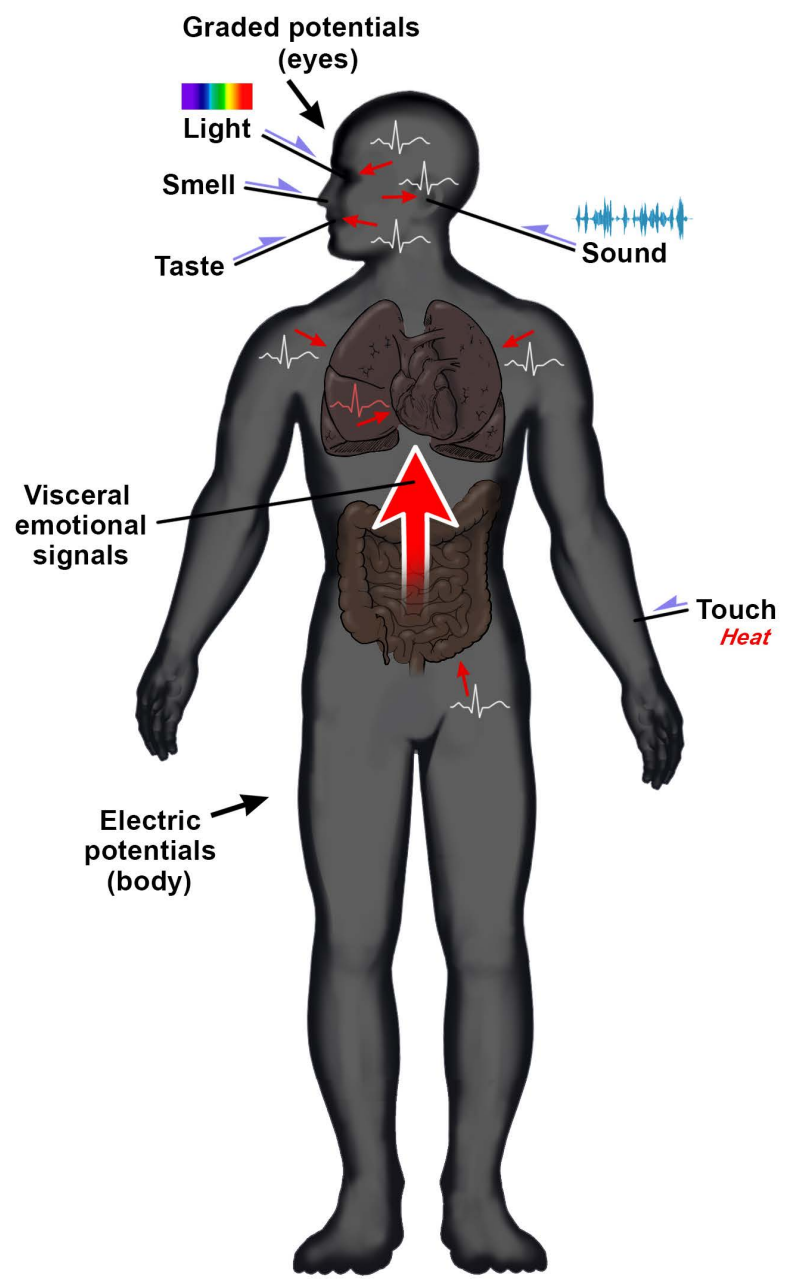

The Body's Internal 3D Default Space

Figure 1. The internal space. The internal "dark" space of the default space forms the foundation of conscious experience. We have termed this foundation the dark space, as it is subconscious and normally only perceived when it is filled with sensory perceptions. This space automatically defaults to the physical characteristics of external space with identical dimensions of time, xyz-space, and orientation. Stimuli from the external world are filled into this "skeleton", allowing for optimal recreation and representation of the external world within the mind [10].

and electrical membrane potentials [6]. We have proposed that the thalamus coordinates processed sensory information from the cortex, synchronizes this processed information with the sensory organs, integrates the senses seamlessly, and fills in this information within this default space [6]. The thalamus and corticothalamic feedback loops have a central role in the default space model with scientific support from various studies as well as being components of other consciousness models [12] [13]. The default space model posits the thalamus as the central hub of the default space with 50 or more integrated nuclei coordinated by the reticular nucleus [6]. This central hub acts as a tiny "observer" of the large electro-chemical space formed by the trillions of cells of the body. The 
processing of the bodily signals through the corticothalamic feedback loops is not complete until these signals become filtered, amplified, and integrated by the many cortical modules.

The default space is always active in a conscious person and exists in an idling state even without sensory input [6]. This idling state is maintained by baseline neural oscillatory activity from salient networks such as the default mode network and brainstem [8]. The oscillations occur throughout the entire body and are dominated by the alpha, beta, and gamma frequencies, however, range from slow to ultrafast [14]. These self-sustaining oscillations of feedforward and feedback information synchronize the sensory organs and cortical counterparts [4]. The synchronized information is filled into the intra-personal space; therefore, the brain and body function together in a unified fashion [2]. This global dynamic network between the central and peripheral nervous systems: 1) is highly organized, 2) includes the transmission of very specific electrical and chemical signals that are self-perceived internally, and 3) replicates the external and internal world [4].

Although the external world is recreated internally, this replication is not a sole result of exteroceptive and interoceptive sensory stimuli. The final simulation of the external world is heavily influenced by attention, memory, and other executive functions [6]. The thalamus plays an important role in the selective attention of sensory information [15], and thalamic injury can lead to severe attentional deficits [16]. Due to the thalamus being the central hub in coordinating sensory integration into the default space, attention to certain sensation modulates what information is integrated into consciousness [6]. Thalamocortical oscillations adaptively resonate with sensory organs in a format organized by the thalamus so that those sensory organs selectively paid attention to rise to awareness [6]. This attentional gating occurs through the thalamic reticular nucleus [17] [18], directing attention via an internal attentional "searchlight" [19]. Through inhibiting or disinhibiting certain thalamic relay cells, the thalamic reticular nucleus may control which sensory organs are synchronized with the cortex [18].

Comatose states are characterized by an absence of arousal and consciousness. Through studying what happens when consciousness is absent, we can better understand what leads to it. The important role of the thalamus in consciousness is demonstrated in the development of coma after thalamic lesion [20]. Thalamic stimulation has been shown to be a possible treatment for comatose states through case studies of resulting responsive behavior [21] [22]. Recently, non-invasive ultrasound pulsation techniques have proven successful in stimulating the thalamus leading to substantial improvement of patients along the Coma Recovery Scale [23]. This effect is believed to act via modulation of thalamocortical communication [24] and has been shown to speed anesthetic recovery in animal models [25]. During anesthetized states, there is reduced corticothalamic connectivity [26] and thalamic metabolism and blood flow are reduced [27]. 


\section{Theoretical Advancement}

Since our main article laying the foundations of the default space model was published in 2015 [6], we have continued to develop new concepts within the scope of the model as well as improve on its principles in order to further explain and describe the role of the default space in memory, cognition, creativity, emotions, meditation, sleep, and other physiological functions. We have outlined the main advances here in order to update readers following our theory as well as provide increasing insight into our model.

\section{Lateral Inhibition}

Based on recent articles on an elusive physiological phenomenon, sensory lateral inhibition, the functional role of this phenomenon in the emergence of perception has been elucidated [2] [3]. We have explained how fast gamma oscillatory communications between peripheral receptors such as photoreceptors exhibit lateral inhibition, thus allowing the corticothalamic networks to "see" the retina (or other sensory organ) as if it were a 3D "Smart Screen" [3]. The currently accepted view in science is that this universal physiology is local and "lateral" [28]. When we speak of lateral inhibition, we do not mean in the exact commonly used sense indicating cells inhibiting physically adjacent cells, however, cortical inhibitions of sensory organ cells that are "adjacent" through synchronization. Although the cortex is not physically adjacent to the sensory organs, it is functionally adjacent due to the synchronization of said cortex with such sensory organs via membrane potential oscillations [6]. This allows communication at near the same time interval as if the cells where physically adjacent. This fast time interval is possible at such separated distances due to the speed of charge transmission and the frequency of the oscillations. We do however note that receptors synchronized with the cortex laterally inhibit its physical neighbors, although the main source of processing determining how cells are inhibited comes from the cortex. This can lead to a magnification of sensory signals, and/or a filtering of unwanted data [3].

We propose that such lateral inhibition occurs among all sensory organs as a main component of perception. In the case of vision, all retinal cells oscillate with the visual and parietal cortices creating a 3D visual space [2]. This creates the infrastructure needed for the integration of stimuli received by all photoreceptors among the retina. In this way, the visual cortex binds its information of color, distance, and position synchronously with the photoreceptors through lateral inhibition, allowing the retina to near instantly integrate new visual stimuli into this visual infrastructure [2]. Through this mechanism, a well mapped and calibrated internal visual space is maintained. We have proposed how this infrastructure is projected onto the retina itself with depth encoded in the disc layers of the photoreceptors [2]. This encoding provides a third dimension to an otherwise flat visual image. The depth is calibrated along the $\sim 1000$ discs in each photoreceptor [29]. Each photoreceptor sees a portion of the entire visual image 
similar to an individual pixel on a screen (Figure 2).

An initially weak visual image is received by the ON-center photoreceptors. The information from the ON-center photoreceptors becomes magnified in graded potentials onto OFF-center photoreceptors with strong amplification and modification from the cortex based on its current expectation of the scene [2]. This image on the OFF-center is that image we actually see which is projected to the brain to be integrated into the default space via the retino-geniculo-cortical oscillations [2]. The same mechanism is active in faculties used to hear, taste, smell and touch [3]. Sensations perceived may be derived directly from actual physical information such as light and sound [30]; however, the dominant perceptions are cortically processed sensory information at the site of the receptors where sensory transduction originally occurs [4]. The information for these top-down perceptions are amplified, processed sensory stimuli combined with perceptive expectations derived from memory, creative, and executive mental functions.

The neural correlates of the senses are anatomically well defined, but understanding how biological membrane potential oscillations modify internal functional sensory processing requires examination of lateral inhibition at the site of initial stimulus. The default space is dynamically active and its precise organization allows cortical centers to parallel process incoming signals from the receptor

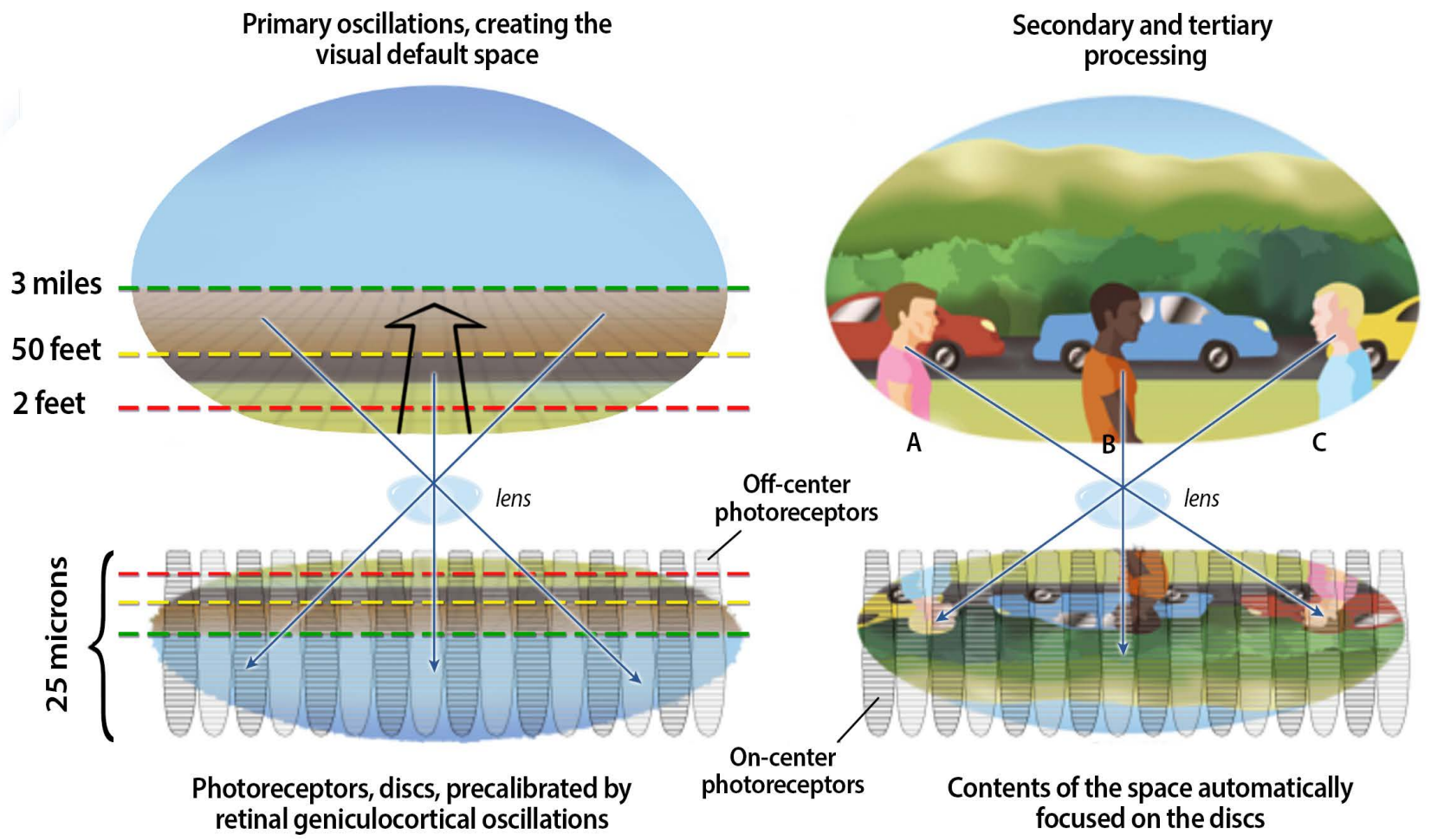

Figure 2. Calibration of photoreceptors and discs in the retina. Retino-geniculo-cortical oscillations pre-calibrate the retina for distance, depth, color, and motion. These oscillations are the foundation of visual perception, and are required for additional processing to occur. This foundation which creates the dark visual space allows the contents of the external space to automatically be focused on the discs, allowing advanced visual perception to take place [5]. 
site and "views" these as proxies of external space, objects, music, etc. [31]. All this transpires in relationship to thalamus, with the coordinates of anterior, posterior, right, left, superior or inferior being oriented in respect to the thalamus and the surrounding default space [31]. This space is precisely defined by the micro-calibration of the retina for visual space and the less precisely defined non-visual space calibrated by the cochlea [31]

\section{Visual Consciousness}

We have asserted with support from studies on anesthetics that oscillations in the gamma frequency range $(30-200 \mathrm{~Hz})$ form the foundations of consciousness [32]. However, alpha oscillations are the dominant frequency range in the brain and fundamental to central nervous system function [33]. We assert the main importance of these frequency ranges in the formation of the visual and non-visual internal space.

A great deal of consciousness research has been conducted in relation to vision, assisting us in understanding the nature of mind and its predominantly visual memory, cognition, awareness and creativity [34] [35] [36]. Our visual theory is structured on the thalamus which is the traffic controller of the circular information flow between and among peripheral and central neural networks, integrating and interfacing bottom-up and top-down parallel processes [37]. We have proposed that visually associated cortices such as the striate cortex power the horizontal and amacrine cells of the retina through the retino-geniculo-cortical oscillations, allowing the retina to quickly processes, amplify, and filter incoming visual stimuli [2]. Based on informational analysis of retinal and thalamic action potentials [38] [39], we have proposed that $~ 93 \%$ of visual information present on the retina originates from cortical areas responsible for memory, spatial, executive, and many other functions [5]. These cortical areas transmit information on depth, space, location, distance, color, and motion to the retina via lateral inhibition [2].

The initiation of a conscious experience of a visual scene begins when visual sensory input is transduced on the retina [40]. Prior to a specific visual datum being received, on-center and off-center photoreceptors of the retina are synchronized with the cortex by retino-geniculo-cortical oscillatory activity. This activity oscillates at a default gamma frequency $(30-200 \mathrm{mhz})$ and creates coherence between the photoreceptors, thalamus, and relevant cortical areas [2]. The oscillations allow the photoreceptors to create a default visual space which is pre-existing, meaning it exists continuously regardless of incoming sensory data and is embryonically developed. As we have described in our explanation of lateral inhibition, external photons land on the on-center photoreceptors where they go through amplification and lateral inhibition via the cortex [2]. The final imagery experienced exists on the off-center photoreceptors, thereby providing conscious vision of the objects in sight [2]. This activity throughout the whole retina creates the visual field of internal space (Figure 3 ). 


\section{The 3D Default Space Visual Fields}
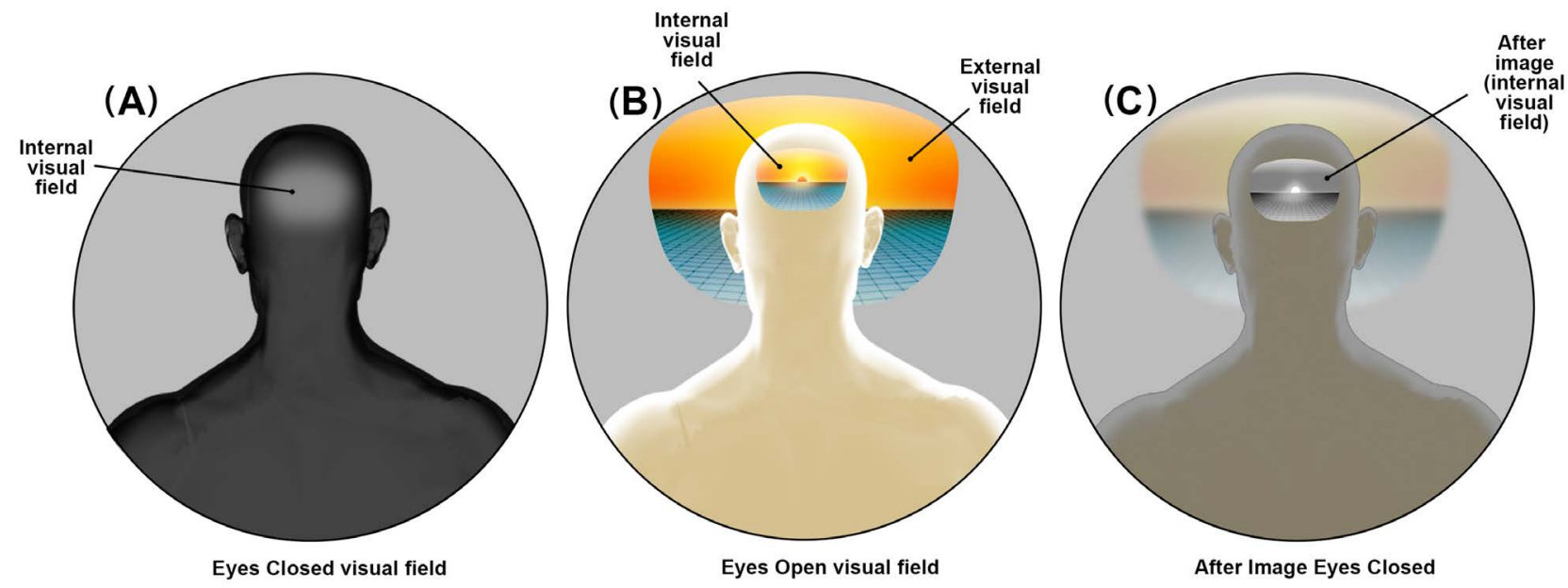

Figure 3. Visual fields of the default space. (A) Appearing dark and with fuzzy boarders, the intra-personal visual space allows for internal vision that forms the default foundation for external visual imagery to be replicated within. Baseline retino-geniculo-cortical oscillations maintain the experience of external visual space; (B) The seamless integration of binocular vision when viewed from behind the eyes respective to the thalamus. The internal visual replication of the external visual field contains precisely calibrated distances and scales; (C) The after image of a visual scene viewed upon closing the eyes demonstrates that we carry a replication of the external world within our minds even without present sensory input. The after image remains when the eyes close, however, it follows the viewer when their orientation changes, breaking away from the orientation of external space. This demonstrates that the seemingly external image is truly internal [41].

\section{Respiration and Membrane Potentials}

The basic biology of membrane excitability necessary for the membrane potential oscillatory activity behind consciousness has an ancient evolutionary basis. All biological cells have evolved homeostatic mechanisms of energy-consuming import-export of ions and molecules and the channel proteins utilized by animal organisms first evolved 1 billion years ago [42]. We further the notion that the unique cellular phenomenon of membrane excitability underlies many aspects of animal psychology by asserting the harmonic and organized oscillatory systems based on the changes in individual cell membranes are the foundation of sentience. Temporal sequencing of neuronal events across brain systems results in awareness and the charge of each cell is the primordial building block to organism-level awareness [42]. We draw additional support for our concepts of membrane oscillatory activity from the arguments by researchers that synchronization of neuronal spikes through ion waves [43] [44] [45] and ephaptic communications [46] are likely mechanisms for explaining the unification of cognitive processes [47].

We have proposed how the unified consciousness we experience is energized by breathing [4]. We have deduced through studies on the electron transport chain, membrane potentials, and ATP formation [48] that during inspiration, electrons are released into the body that raise the membrane potentials of cells throughout the body [4]. Via the electron transport chain, a much greater amount of ATP (32 molecules) is produced per molecule of glucose when suffi- 
cient oxygen is present versus when a lack of oxygen exists (2 molecules) [49]. "This charging" of membrane potentials occurs in part through the ATP powering of potassium channels which pump potassium ions across the membrane, resulting in a more polarized membrane [50]. Our assertion is that respiration influences brain activity, the autonomic nervous system, blood pressure, heart activity, homeostatic physiology, and behavior.

We further propound that beyond the ATP powered membrane potential hyperpolarization, respiration creates electrons via slowly adapting stretch receptors which are able to penetrate bodily tissues and further power ion channels to hyperpolarize membranes [4]. Our main assertion on respiration is that respiration is responsible for the dynamic polarization fluctuations observed in cell membranes [4]. These fluctuations are the source of brain waves which have shown correlation to respiration [51]. Furthermore, the autonomic nervous system is modulated by membrane potential which can itself be modulated by respiration [52]. By synchronizing respiration with heartbeat (cardiorespiratory coherence) and lowering the ratio of heartbeats to breaths, one can induce a relaxation response, which has been demonstrated to decrease oxygen consumption, blood pressure, and sympathetic reactivity to stress [53]. We have proposed that this decrease in sympathetic reactivity occurs via the increases in neuronal membrane potentials described [54].

\section{Emotion and the Mind-Body Response}

Specific patterns of breathing are intimately associated with specific emotions [55] based on predictable oscillations in the mind [4]. Breathing has demonstrated the capability to modulate fear, arousal, and cognitive states while sleep, emotions, exercise, hypoxia, and hypercapnia have been shown to modulate breathing [56]. Deep breathing techniques such as "Pranayama" have been experimentally shown to aid in many negative emotional modulations of mind by breaking the breathing patterns associated with the troubling emotions [57] [58] [59]. Meditation techniques have been demonstrated to attenuate symptoms of depression, anxiety, negative coping and affect, self-hostility, and even post-traumatic stress disorder [53] [60]. The mind responds to the physiological state the body is under, of which breathing is a main part, by undergoing the emotion(s) associated with that physiological state(s). We have labeled this brain-body unity phenomenon of the state of the body affecting the mind and vice-versa the "mind-body response" [54]. The practical applications of this concept can be a powerful influence over one's daily mental and physical health.

The mind body response asserts that in a snowball like effect, controlling one's breathing pattern leads the mind to experience the emotion associated with that breathing pattern, which in turn influences the breathing pattern automatically, further strengthening the emotion of the breathing pattern in the mind [54]. A similar effect is seen in expressions of emotion which are readily communicated to peers through facial expression. The mind body response hypothesis predicts 
what numerous research experiments have shown; that forced facial expressions are reflected in the state of the mind. Forced laughter and smiling substantially improves mood in participants [61]. As we have described how the external world is represented in internal space, mindfulness of breathing can make a person more aware of this internal space and feel alive within a large limitless expanse of space [7]. Without knowledge of this internal world, one may feel it is the external world that they experience as limitless and that it is a part of them. Various levels of personality and emotional disorders may further lead to more problematic psychological and psychiatric disorders through the misidentification of the external world with the internal. This characteristic of the default space needs to be recognized and taken into consideration when evaluating and treating mental disorders.

\section{Mediation Experiences}

Many studies on meditation has unequivocally shown it to facilitate the ability to remain calm under stressful situations, control one's emotions, and gain a better understanding of the "self" [62] [63] [64] [65]. Meditation has been shown to activate the prefrontal cortex and increase levels of free glutamate in the brain, stimulating increases in serotonin [66] [67]. In addition, many other brain regions are activated or altered during meditation, specifically in respect to our work, the default mode network is altered [68]. Meditation associated physiological changes such as reduction in respiration and heart rate facilitate positive feelings, consolidation of new memories, and learning [69] [70].

There are certain experiences that occur during various mediation techniques that support notions of the default space model. Those that practice transcendental meditation report experiences that transcend normal states of being which are characterized by unbounded space and time [71]. We have proposed that this unbounded space experienced is indeed the default space which is not normally apparent in normal states of consciousness [7]. Although we are consciously unaware of this 3D space, our unconscious mind uses it as the framework for reproduction of the external world [9]. Through the intense introspection that occurs during meditation, one can achieve a deeper interoceptive awareness that not only leads to a more vivid perception of visceral feelings and emotions [72], but also the boundless internal expanse of the default space.

\section{Mechanism of Out of Body Experiences}

We have proposed how the cornerstones of our intrapersonal space are the retino-geniculo-cortical oscillations [2] and the corticothalamic oscillations [6]. The brain receives multisensory inputs and binds these inputs into a neural map that serves to simulate the external world within [6]. Out of body experiences (OBE) are a type of autoscopic phenomenon, a visual hallucination that creates the perception of disembodiment or existing outside of one's body [73]. During an OBE, a person can feel that in addition to their sensory perspective, their feel- 
ings, thoughts, and emotions are also occurring from this outside visuo-spatial perspective [74]. The person may experience possession of a duplicate body from their hallucinatory perspective while still feeling ownership of the body they see apart from their perspective as if looking at a reflection [75].

Corticothalamic oscillations link all parts of our sensory experience with an intrapersonal space that replicates the external space [2] [76]. The thalamus integrates sensory information with visuo-spatial and executive information with the cortex, creating a unified conscious experience which is structured on the recreation of external space [2]. We have proposed that if abnormal cortical activity occurs in this process, then the thalamus may recreate an external space that has an inaccurate perspective [9]. Due to the nature of retino-geniculo-oscillations synchronizing the cortex with the retina [2], we have proposed that if abnormal activity were processed through these oscillations, this information would be projected onto the retina leading to the experience of this alternate perspective [9]. Due to the thalamus being the hub of sensory integration as well as space, time, body location, and sense of self, alterations in some if this information could lead to an overall alteration once the many aspects of a conscious experience have been unified [9].

\section{Conclusions}

The default space model identifies an internal 3D neural space which is the foundation and structure of conscious experience. Membrane potential oscillations from the body and brain are coordinated, synchronized, and harmonized by the thalamus so that all internally and externally derived sensory information is filled into this 3D neural space in order to simulate the external world within. Top down dominated synchronization of the sensory organs with cortical structures brings these cortical areas to the sensory organs allowing sensory stimuli to be processed at the sensory organ site.

In this article we have reviewed the updates of our dynamic global theory that is supported by clinical studies, first person observations, and etiologies of clinical deficits. Aside from the nature of the origin of reality itself, consciousness is the most mysterious concept known to man. Understanding how it arises and how it exists in our universe would not only greatly benefit the fields of neuroscience, artificial intelligence, and general health, but also holds untold possibilities for our future not only as human beings, but as conscious entities.

\section{References}

[1] Young, R.M. (1996) The Mind-Body Problem. Companion to the History of Modern Science. Taylor and Francis, Milton Park.

[2] Jerath, R., et al. (2016) How Lateral Inhibition and Fast Retinogeniculo-Cortical Oscillations Create Vision: A New Hypothesis. Medical Hypotheses, 96, 20-29. https://doi.org/10.1016/j.mehy.2016.09.015

[3] Jerath, R., et al. (2017) Sensory Consciousness Is Experienced through Amplification of Sensory Stimuli via Lateral Inhibition. World Journal of Neuroscience, 7, 
244-256. https://doi.org/10.4236/wjns.2017.73020

[4] Jerath, R., et al. (2017) The Dynamic Role of Breathing and Cellular Membrane Potentials in the Experience of Consciousness. . World Journal of Neuroscience, 7, 66-81. https://doi.org/10.4236/wjns.2017.71007

[5] Jerath, R., Cearley, S.M., Barnes, V.A. and Jensen, M. (2018) Micro-Calibration of Space and Motion by Photoreceptors Synchronized in Parallel with Cortical Oscillations: A Unified Theory of Visual Perception. Medical Hypotheses, 110, 71-75. https://doi.org/10.1016/j.mehy.2017.11.005

[6] Jerath, R., Crawford, M.W. and Barnes, V.A. (2015) A Unified 3D Default Space Consciousness Model Combining Neurological and Physiological Processes That Underlie Conscious Experience. Frontiers in Psychology, 6, Article ID: 1204. https://doi.org/10.3389/fpsyg.2015.01204

[7] Jerath, R., et al. (2016) Meditation Experiences, Self, and Boundaries of Consciousness. International Journal of Complementary \& Alternative Medicine, 4, Article ID: 00105.

[8] Jerath, R. and Crawford, M.W. (2015) Layers of Human Brain Activity: A Functional Model Based on the Default Mode Network and Slow Oscillations. Frontiers in Human Neuroscience, 9, 248. https://doi.org/10.3389/fnhum.2015.00248

[9] Jerath, R., et al. (2016) Functional and Neural Mechanisms of Out-of-Body Experiences: Importance of Retinogeniculo-Cortical Oscillations. World Journal of Neuroscience, 6, 287-302. https://doi.org/10.4236/wjns.2016.64032

[10] Jerath, R. and Jensen, M. (2016) Meditation Experiences, Self, and Boundaries of Consciousness. International Journal of Complementary \& Alternative Medicine, 4, Article ID: 00105

[11] Mader, S.S. (2000) Human Biology. McGraw-Hill, New York.

[12] Edelman, G.M. (2004) Wider than the Sky: The Phenomenal Gift of Consciousness. Yale University Press, New Haven.

[13] Baars, B.J., Franklin, S. and Ramsoy, T.Z. (2013) Global Workspace Dynamics: Cortical "Binding and Propagation" Enables Conscious Contents. Frontiers in Psychology, 4, 200. https://doi.org/10.3389/fpsyg.2013.00200

[14] Moran, L.V. and Hong, L.E. (2011) High vs Low Frequency Neural Oscillations in Schizophrenia. Schizophrenia Bulletin, 37, 659-663. https://doi.org/10.1093/schbul/sbr056

[15] Tokoro, K., et al. (2015) Thalamus and Attention. Brain and Nerves, 67, 1471-1480.

[16] Van der Werf, Y.D., et al. (2003) Deficits of Memory, Executive Functioning and Attention Following Infarction in the Thalamus; A Study of 22 Cases with Localised Lesions. Neuropsychologia, 41, 1330-1344. https://doi.org/10.1016/S0028-3932(03)00059-9

[17] McAlonan, K., Brown, V.J. and Bowman, E.M. (2000) Thalamic Reticular Nucleus Activation Reflects Attentional Gating during Classical Conditioning. Journal of Neuroscience, 20, 8897-8901. https://doi.org/10.1523/JNEUROSCI.20-23-08897.2000

[18] Lam, Y.W. and Sherman, S.M. (2011) Functional Organization of the Thalamic Input to the Thalamic Reticular Nucleus. Journal of Neuroscience, 31, 6791-6799. https://doi.org/10.1523/JNEUROSCI.3073-10.2011

[19] Crick, F. (1984) Function of the Thalamic Reticular Complex: The Searchlight Hypothesis. Proceedings of the National Academy of Sciences of the United States of America, 81, 4586-4590. https://doi.org/10.1073/pnas.81.14.4586 
[20] Bjornstad, B., et al. (2003) Paroxysmal Sleep as a Presenting Symptom of Bilateral Paramedian Thalamic Infarctions. Mayo Clinic Proceedings, 78, 347-349. https://doi.org/10.4065/78.3.347

[21] Schiff, N.D., et al. (2007) Behavioural Improvements with Thalamic Stimulation after Severe Traumatic Brain Injury. Nature, 448, 600-603. https://doi.org/10.1038/nature06041

[22] Shah, S.A. and Schiff, N.D. (2010) Central Thalamic Deep Brain Stimulation for Cognitive Neuromodulation-A Review of Proposed Mechanisms and Investigational Studies. European Journal of Neuroscience, 32, 1135-1144. https://doi.org/10.1111/j.1460-9568.2010.07420.x

[23] Monti, M.M., et al. (2016) Non-Invasive Ultrasonic Thalamic Stimulation in Disorders of Consciousness after Severe Brain Injury: A First-in-Man Report. Brain Stimulation: Basic, Translational, and Clinical Research in Neuromodulation, 9, 940-941. https://doi.org/10.1016/j.brs.2016.07.008

[24] Schiff, N.D. (2010) Recovery of Consciousness after Brain Injury: A Mesocircuit Hypothesis. Trends in Neurosciences, 33, 1-9.

https://doi.org/10.1016/j.tins.2009.11.002

[25] Bystritsky, A., et al. (2011) A Review of Low-Intensity Focused Ultrasound Pulsation. Brain Stimulation, 4, 125-136.

[26] White, N.S. and Alkire, M.T. (2003) Impaired Thalamocortical Connectivity in Humans during General-Anesthetic-Induced Unconsciousness. Neuroimage, 19, 402-411. https://doi.org/10.1016/S1053-8119(03)00103-4

[27] Alkire, M.T. and Miller, J. (2005) General Anesthesia and the Neural Correlates of Consciousness. Progress in Brain Research, 150, 229-244. https://doi.org/10.1016/S0079-6123(05)50017-7

[28] Yantis, S. (2014) Sensation and Perception. Worth Publishers, New York.

[29] Burgoyne, T., et al. (2015) Rod Disc Renewal Occurs by Evagination of the Ciliary Plasma Membrane That Makes Cadherin-Based Contacts with the Inner Segment. Proceedings of the National Academy of Sciences, 112, 15922-15927. https://doi.org/10.1073/pnas.1509285113

[30] Herrero, M.T., Barcia, C. and Navarro, J.M. (2002) Functional Anatomy of Thalamus and Basal Ganglia. Child's Nervous System, 28, 386-404. https://doi.org/10.1007/s00381-002-0604-1

[31] Ravinder Jerath, M.W.C. and Barnes, V.A. (2015) A Unified 3D Default Space Consciousness Model Combining Neurological and Physiological Processes That Underlie Consciuos Experience. Frontiers in Psychology, 6, 1204.

[32] Plourde, G. and Arseneau, F. (2017) Attenuation of High-Frequency (30-200 Hz) Thalamocortical EEG Rhythms as Correlate of Anaesthetic Action: Evidence from Dexmedetomidine. BJA: British Journal of Anaesthesia, 119, 1150-1160.

[33] Klimesch, W. (2012) Alpha-Band Oscillations, Attention, and Controlled Access to Stored Information. Trends in Cognitive Sciences, 16, 606-617. https://doi.org/10.1016/j.tics.2012.10.007

[34] Gregory, R.L. (1997) Knowledge in Perception and Illusion. Philosophical Transactions of the Royal Society of London. Series B: Biological Sciences, 352, 1121-1127. https://doi.org/10.1098/rstb.1997.0095

[35] Gregory, R.L. (1970) The Intelligent Eye. Weidenfeld and Nicolson, London.

[36] Gibson, J.J. (1966) The Senses Considered as Perceptual Systems. Houghton Mifflin Boston. 
[37] Guillery, R.W. and Sherman, S.M. (2002) Thalamic Relay Functions and Their Role in Corticocortical Communication. Neuron, 33, 163-175. https://doi.org/10.1016/S0896-6273(01)00582-7

[38] Sherman, S.M. (2005) Thalamic Relays and Cortical Functioning. Progress in Brain Research, 149, 107-126. https://doi.org/10.1016/S0079-6123(05)49009-3

[39] Wang, X., Sommer, F.T. and Hirsch, J.A. (2011) Inhibitory Circuits for Visual Processing in Thalamus. Current Opinion in Neurobiology, 21, 726-733. https://doi.org/10.1016/j.conb.2011.06.004

[40] Galambos, R., et al. (2005) The Human Retinal Functional Unit. International Journal of Psychophysiology, 57, 187-194. https://doi.org/10.1016/j.ijpsycho.2005.01.013

[41] Jerath, R. and Jensen, M. (2016) Visual Fields of the Default Space. Int J Complement Alt Med: Meditation Experiences, Self, and Boundaries of Consciousness.

[42] Cook, N.D., Carvalho, G. and Damasio, A. (2014) From Membrane Excitability to Metazoan Psychology. Trends in Neurosciences, 37, 698-705. https://doi.org/10.1016/j.tins.2014.07.011

[43] Aur, D. and Jog, M.S. (2010) Neuroelectrodynamics: Understanding the Brain Language. IOS Press, Amsterdam.

[44] Black, J.A. and Waxman, S.G. (2013) Noncanonical Roles of Voltage-Gated Sodium Channels. Neuron, 80, 280-291. https://doi.org/10.1016/j.neuron.2013.09.012

[45] Fields, R.D. (2009) The Other Brain. Simon \& Schuster, New York.

[46] Anastassiou, C.A. (2011) Ephaptic Coupling of Cortical Neurons. Nature Neuroscience, 14, 217-223. https://doi.org/10.1038/nn.2727

[47] von der Malsburg, C. (2010) Dynamic Coordination in the Brain: From Neuron to Mind. MIT Press, Cambridge. https://doi.org/10.7551/mitpress/9780262014717.001.0001

[48] Pittman, R.N. (2011) The Circulatory System and Oxygen Transport.

[49] Guyton, A.C. and Hall, J.E. (2000) Textbook of Medical Physiology. 10th Edition, W.B. Saunders, Philadelphia.

[50] Laskowski, M., et al. (2016) What Do We Not Know about Mitochondrial Potassium Channels? Biochimica et Biophysica Acta (BBA)-Bioenergetics, 1857, 1247-1257. https://doi.org/10.1016/j.bbabio.2016.03.007

[51] Jerath, R., et al. (2006) Physiology of Long Pranayamic Breathing: Neural Respiratory Elements May Provide a Mechanism That Explains How Slow Deep Breathing Shifts the Autonomic Nervous System. Medical Hypotheses, 67, 566-571. https://doi.org/10.1016/j.mehy.2006.02.042

[52] Jerath, R. and Crawford, M.W. (2015) How Does the Body Affect the Mind? Role of Cardiorespiratory Coherence in the Spectrum of Emotions. Advances in Mind-Body Medicine, 29, 4-16.

[53] Esch, T., Fricchione, G.L. and Stefano, G.B. (2003) The Therapeutic Use of the Relaxation Response in Stress-Related Diseases. Medical Science Monitor, 9, RA23-RA34.

[54] Jerath, R., Barnes, V.A. and Crawford, M.W. (2014) Mind-Body Response and Neurophysiological Changes during Stress and Meditation: Central Role of Homeostasis. Journal of Biological Regulators \& Homeostatic Agents, 28, 545-554.

[55] Homma, I. and Masaoka, Y. (2008) Breathing Rhythms and Emotions. Experimental Physiology, 93, 1011-1021. https://doi.org/10.1113/expphysiol.2008.042424 
[56] Ramirez, J.M., et al. (2012) The Cellular Building Blocks of Breathing, in Comprehensive Physiology. John Wiley \& Sons, Inc., Hoboken.

[57] Nemati, A. (2013) The Effect of Pranayama on Test Anxiety and Test Performance. International Journal of Yoga, 6, 55-60. https://doi.org/10.4103/0973-6131.105947

[58] Gupta, P.K., Kumari, M., Deo, R. and Anuloma-Viloma, J. (2010) Pranayama and Anxiety and Depression among the Aged. Journal of the Indian Academy of Applied Psychology, 36, 159-164.

[59] Bhargava, R., Gogate, M. and Mascarenhas, J. (1988) Autonomic Responses to Breath Holding and Its Variations Following Pranayama. Indian Journal of Physiology and Pharmacology, 32, 257-264.

[60] Rosenzweig, S., et al. (2007) Mindfulness-Based Stress Reduction Is Associated with Improved Glycemic Control in Type 2 Diabetes Mellitus: A Pilot Study. Alternative Therapies in Health and Medicine, 13, 36-38.

[61] Neuhoff, C.C. and Schaefer, C. (2002) Effects of Laughing, Smiling, and Howling on Mood. Psychological Reports, 91, 1079-1080.

[62] van der Zwan, J.E., et al. (2015) Physical Activity, Mindfulness Meditation, or Heart Rate Variability Biofeedback for Stress Reduction: A Randomized Controlled Trial. Applied Psychophysiology and Biofeedback, 40, 257-268.

https://doi.org/10.1007/s10484-015-9293-x

[63] Rod, K. (2015) Observing the Effects of Mindfulness-Based Meditation on Anxiety and Depression in Chronic Pain Patients. Psychiatria Danubinad, 27, S209-S211.

[64] Zeidan, F., et al. (2014) Neural Correlates of Mindfulness Meditation-Related Anxiety Relief. Social Cognitive and Affective Neuroscience, 9, 751-759. https://doi.org/10.1093/scan/nst041

[65] Goyal, M., et al. (2014) Meditation Programs for Psychological Stress and Well-Being: A Systematic Review and Meta-Analysis. JAMA Internal Medicine, 174, 357-368.

[66] Guggisberg, A.G., et al. (2011) The Neural Basis of Event-Time Introspection. Consciousness and Cognition, 20, 1899-1915. https://doi.org/10.1016/j.concog.2011.03.008

[67] Newberg, A.B. and Iversen, J. (2003) The Neural Basis of the Complex Mental Task of Meditation: Neurotransmitter and Neurochemical Considerations. Medical Hypotheses, 61, 282-291. https://doi.org/10.1016/S0306-9877(03)00175-0

[68] Garrison, K.A., et al. (2015) Meditation Leads to Reduced Default Mode Network Activity beyond an Active Task. Cognitive, Affective, \& Behavioral Neuroscience, 15, 712-720. https://doi.org/10.3758/s13415-015-0358-3

[69] Barnes, V.A., et al. (1999) Acute Effects of Transcendental Meditation on Hemodynamic Functioning in Middle-Aged Adults. Psychosomatic Medicine, 61, 525-531. https://doi.org/10.1097/00006842-199907000-00017

[70] Deshmukh, V.D. (2006) Neuroscience of Meditation. Scientific World Journal, 6, 2239-2253. https://doi.org/10.1100/tsw.2006.353

[71] Travis, F. (2014) Transcendental Experiences during Meditation Practice. Annals of the New York Academy of Sciences, 1307, 1-8. https://doi.org/10.1111/nyas.12316

[72] Cali, G., et al. (2015) Investigating the Relationship between Interoceptive Accuracy, Interoceptive Awareness, and Emotional Susceptibility. Frontiers in Psychology, 6, 1202. https://doi.org/10.3389/fpsyg.2015.01202

[73] Anzellotti, F., et al. (2011) Autoscopic Phenomena: Case Report and Review of Literature. Behavioral and Brain Functions, 7, 2. 
https://doi.org/10.1186/1744-9081-7-2

[74] Blanke, O., et al. (2004) Out-of-Body Experience and Autoscopy of Neurological Origin. Brain, 127, 243-258. https://doi.org/10.1093/brain/awh040

[75] Heydrich, L. and Blanke, O. (2013) Distinct Illusory Own-Body Perceptions Caused by Damage to Posterior Insula and Extrastriate Cortex. Brain, 136, 790-803. https://doi.org/10.1093/brain/aws364

[76] Niculescu, D. and Lohmann, C. (2014) Gap Junctions in Developing Thalamic and Neocortical Neuronal Networks. Cerebral Cortex, 24, 3097-3106.

https://doi.org/10.1093/cercor/bht175 